\section{Choosing a Cantilever for In Situ Atomic Force Microscopy}

John T. Woodward

Biotechnology Division, National Institute of Standards and Technology

John.Woodward@nist.gov

What is the best cantilever for intermittent contact mode (often called Tapping Mode $\left.{ }^{T M}\right)^{12}$ atomic force microscope (AFM) imaging under water? This is a question I hear often and one that recently generated some interesting discussion on an AFM newsgroup (more on the newsgroup below). The ability of the AFM to image samples in physiologically relevant environments has made it a popular technique in the biological sciences. However, because scanning the AFM tip in contact mode easily perturbs many biological samples, it was the advent of intermittent contact modes that lead to AFM's widespread use in biology. Intermittent contact mode, in which the tip is tapped instead of dragged across the sample, greatly reduces the lateral forces that the tip imparts to the sample and has greatly enhanced the ability of AFM to image soft samples. Yet, it is not a cure-all technique and soft biological samples remain among the most challenging to image. When imaging difficult samples, it often pays to experiment with different imaging conditions, and trying different cantilevers is no exception. The discussion below should aid researchers to experiment more productively by understanding the principles underlying intermittent contact mode imaging.

In intermittent contact mode, the AFM cantilever is oscillated at or near its resonance frequency and brought near the sample surface so that the tip hits (or taps) the surface at the bottom of each oscillation. When the tip hits the surface, the amplitude of the oscillation is reduced, and the magnitude of this amplitude is used as the feedback signal to generate the AFM image. The freely oscillating cantilever behaves as a "damped, driven, harmonic oscillator." Physicists and engineers commonly study this system in mechanics courses. The following discussion is of a qualitative nature, but the interested reader can find detailed equations in many textbooks. ${ }^{3}$

For our purposes there are three properties of the cantilever that we want to consider, the resonance frequency, $f_{0}$, the spring constant, $k$, and the quality factor, $Q$. The spring constant is an intrinsic property of the cantilever that depends on its shape and material properties, while $f_{0}$ and $Q$ depend both on $k$ and the local environment of the cantilever. The spring constant is a measure of how much force is required to bend the cantilever a certain distance. Thus, the spring constant multiplied by the distance the cantilever is deflected by the surface is equal to the force exerted by the tip on the surface. In general, we would like $k$ to be small, so that we can detect the deflection while applying as small a force as possible to the surface to avoid damaging our sample. Cantilevers for tapping in air usually have a high spring constant $(>1 \mathrm{~N} / \mathrm{m})$ because a thin layer of water between the tip and sample creates a meniscus with an adhesion force of $10^{-8}$ $\mathrm{N}$ or more. ${ }^{4}$ To tap on the surface the tip must break free of this meniscus. For a $1 \mathrm{~N} / \mathrm{m}$ cantilever a displacement of $10 \mathrm{~nm}$ will create the required force $\left(1 \mathrm{~N} / \mathrm{m} \times 10^{-8} \mathrm{~m}=10^{-8} \mathrm{~N}\right)$ where as a softer, say $0.01 \mathrm{~N} / \mathrm{m}$, cantilever would require a $1 \mu \mathrm{m}$ displacement. One of the benefits of imaging underwater (or other fluids) is that the meniscus force is gone and we are free to use softer cantilevers and thus can image softer samples. This has opened the door to imaging a wide variety of soft condensed matter and biological samples.

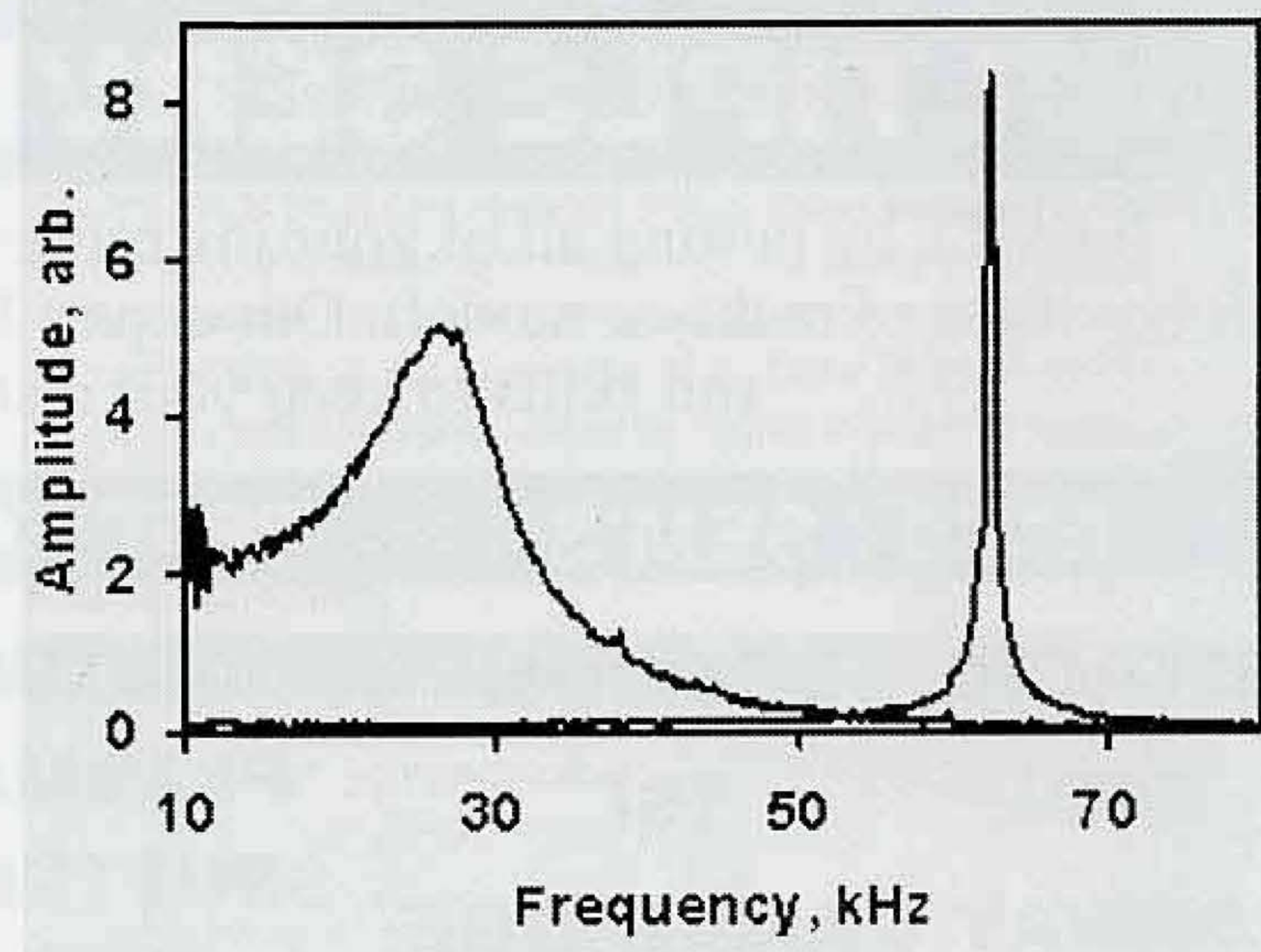

Figure 1. Resonance curves taken in air and water for a magnetically driven, silicon diving board cantilever with a nominal spring constant of $2.8 \mathrm{~N} / \mathrm{m}$. A greater driving force was applied to the cantilever in water. In air, the cantilever has a resonance frequency of $62.4 \mathrm{kHz}$ and $\mathrm{a} \mathrm{Q}$ of 185 . In water, the resonance frequency is 26.5 $\mathrm{kHz}$ and $\mathrm{Q}$ is 3.8. The relatively high underwater resonance frequency allows for reasonably fast scan rates, but the large spring constant may prevent the imaging of softer samples without damage.

Using a cantilever with an appropriate spring constant for a sample is important, but there are still a few issues to consider: The first is the speed with which you can image. If you make a $512 \times 512$ data point image at $1 \mathrm{~Hz}$ scan rate you are collecting data at about $1 \mathrm{kHZ}$ (most instruments take 512 points left to right and 512 points right to left, even if you only view the left to right image). At an absolute minimum you need to tap once for every data point. Thus, the cantilever must have a resonance frequency that is, at an absolute minimum, greater than the data collection rate.

In addition to the requirement of one oscillation per data point, we must consider the more subtle effect of how fast the cantilever responds to the driving force. This is determined in part by the quality factor. The $Q$ of the cantilever relates to the sharpness of the resonance peak when you take a frequency sweep. In air, the cantilever has a relatively high $Q$ and thus a sharp peak, while in water the peak is much broader indicating a lower $Q$ as shown in Figure 1. A higher $Q$ also means a faster response to the driving force. Thus, a cantilever with a high $Q$ will quickly reach its maximum amplitude while one with a low $Q$ may take many oscillations. Ideally, when the tip loses contact with the sample the amplitude of oscillation grows and the feedback of the AFM brings the tip closer to the sample until the amplitude is damped. However, if the amplitude grows slowly, the feedback signal will be small, and the tip may have difficulty following the sample. Therefore, one oscillation per data point is not always enough and the resonance frequency should likely be several times the data collection rate.

As was mentioned above $k, Q$ and $f_{o}$ are not completely independent. All other things being equal, lowering the spring constant also lowers the resonance frequency and the quality factor. Thus, there is a tradeoff between how soft a cantilever you can use and how fast you can image. Many of the samples that I image, such as organic crystals or self-assembled monolayers, are robust enough that I can use the same high- $k$, silicon, diving board cantilevers I use in air. For more delicate samples, the lower $k$, silicon nitride triangular cantilevers are often a better choice. Since the primary 
cause of the damping that lowers $f_{0}$ and $Q$ is drag from the water the more compact cantilevers tend to perform better. Thus the thin legged, $100 \mu \mathrm{m}$ long cantilever is commonly recommended as a good tradeoff between spring constant and scanning speed. There is also work on a new generation of low $k$, high $f_{0}$ cantilevers that are smaller than those currently available. ${ }^{5}$ These hold the promise of high speed imaging with low forces, but will require improvements to the current optical lever systems that detect the tip motion.

At the beginning of this article I mentioned an AFM newsgroup An AFM manufacturer runs the list, but it is open to anyone interested in scanning probe microscopy and the questions and discussions are mostly of a general nature. The group is friendly, informative and treats beginners and experts with equal respect. I recommend it as a resource to anyone in the field. ${ }^{2}$ To subscribe send an email to majordomo@di.com with the following message: subscribe spmdigest your.name@your.domain

Endnotes

Tapping Mode is a registered trademark of Digital Instruments Corporation.

2 The mention of products in this manuscript is not meant as an endorsement by NIST nor as an indication that they are the best available.

${ }^{3}$ A. P. French; Vibrations and Waves; W. W. Norton \& Co.: NewYork, 1971; $77-$ 100.

${ }^{4}$ B. Drake et al., Science 243, 1568-1571 (1989).

${ }^{5}$ M. B. Viani et al., Nature Structural Biology7, 644-647 (2000).

\section{SYMPOSIUM}

New Dimensions in InVivo Imaging (May 30)

The W.M. Keck Laboratory for Biological Imaging and the LOCI Group, University of Wisconsin Madison, in cooperation with the Promega Corporation will present the 2nd Symposium on Biological Imaging. The symposium will highlight new approaches to in vivo imaging, and will cover topics that include fluorescence correlation spectroscopy, in vivo two-photon microscopy, optical coherence tomography, speckle microscopy, small molecule reporters, nanocrystal labeling, and second harmonic imaging. In addition, interactive workshops on automated image analysis and on software for biological visualization will be presented. Contact Ms. Wendy Gelking at Promega Corporation, (608) 274-4330 extension 1194 or wgelking@promega.com

\section{Position Wanted}

Light and electron microscopist with $20+$ years of experience in rubber and plastics. Experienced with P.L.M., Phase, Photomicroscopy, SEM/EDS, TEM, and micro-IR spectroscopy. Other experience includes microchemical testing, dispersion staining, and GC-MS. Developed, implemented, and was responsible for laser safety program in comliance with ANSI Z136.1.

Willing to relocate.

Contact Frank Karl at (330) 773-8330
Comprehensive microscopy information on the web

\section{www.microscopy.info}

Product Guide Laboratory Links

Manufacturers Directory

Microscopy News Product Technology

Career Resources

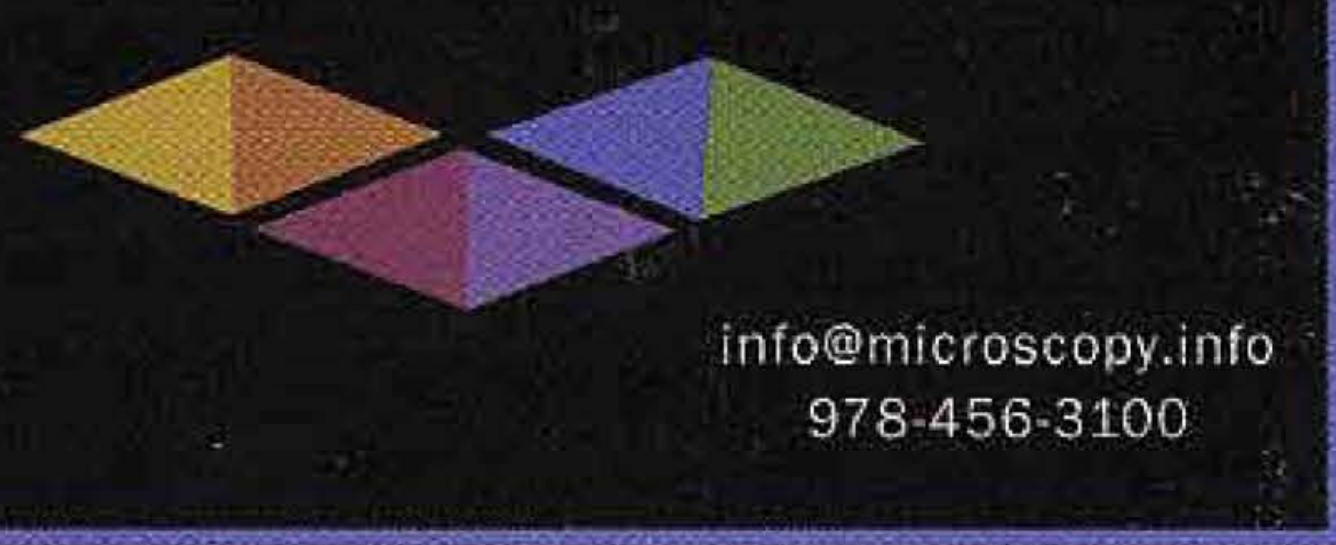

Light Guides \&

Custom Scintillators

from

\section{E. Taylor Engineering, Inc}

Light Guides for Leo/Cambridge model SEMS from $\$ 495$

ITO Gold and Custom Scintillators from \$89

P47 Phospor, YAG, YAP and many others

Highest Quality Engineering

See our web site for special offers on maintenance and recoating specials

M. E. Taylor Engineering. Inc. 21604 Gentry Lane

Brookeville, MD 20833

Phone: (301) 774-6246 